\title{
Comparison of intramuscular progesterone with oral nifedipine for treating threatened preterm labor: A randomized controlled trial
}

\author{
Ladan Haghighi ${ }^{1}$, Mandana Rashidi ${ }^{2}$, Zahra Najmi ${ }^{3 *}$, Homa Homam $^{4}$, Neda Hashemi ${ }^{4}$, \\ Alireza Mobasseri ${ }^{4}$, Yousef Moradi ${ }^{5}$
}

Received: 20 Mar 2017

Published: 6 Sep 2017

\section{Abstract}

Background: Threatened preterm labor (TPL) is the leading cause of hospitalization during pregnancy. Tocolytic agents are the primary therapeutic options for TPL. The aim of this study is to compare intramuscular progesterone with oral nifedipine as a tocolytic agent.

Methods: This randomized controlled trial was carried out in a teaching hospital (Shahid Akbarabadi) in Tehran, Iran, from December 2011 to November 2012. Three hundred and fifteen singleton pregnant women aged >18 yrs at 26-34 weeks' gestation with the diagnosis of threatened preterm labor (TPL) were randomly received either intramuscular progesterone or oral nifedipine for tocolysis. Maternal and neonatal outcomes were then compared between the two interventions. P value less than 0.05 was considered statistically significant. IRCT registration number of this study is IRCT201112198469N1

Results: The success rate of progesterone and nifedipine in treating TPL were $83 \%$ and $82.7 \%$, respectively. There was no significant difference between the two interventions with regard to gestational age at delivery, type of delivery, the time interval until the delivery, birth weight, NICU admission rate and hospital stays. Progesterone administration was associated with lower duration of NICU stay as compared with nifedipine $(0.33 \pm 0.77$ days vs. $1.5 \pm 3.2$ days, $p<0.05)$. None of the two drugs caused any major side effects.

Conclusion: Single dose intramuscular progesterone is as effective as oral nifedipine in treating TPL. It also significantly reduces the NICU stay.

Keywords: Progesterone, Nifedipine, Threatened preterm labor

Copyright $\odot$ Iran University of Medical Sciences

Cite this article as: Haghighi L, Rashidi M, Najmi Z, Homam H, Hashemi N, Mobasseri A, Moradi Y. Comparison of intramuscular progesterone with oral nifedipine for treating threatened preterm labor: A randomized controlled trial. Med J Islam Repub Iran. 2017 (6 Sep);31:56. https://doi.org/10.14196/mjiri.31.56

\section{Introduction}

Threatened preterm labor (TPL) is the leading cause of hospitalization during pregnancy $(1,2)$. Almost $50 \%$ of the women with the diagnosis of TPL would eventually have a preterm delivery. $(1,3)$. Although the majority of these preterm births took place in the developing countries, the rate has recently been increased in the developed world (4). Since the preterm birth is the main cause of neonatal mor-

\section{Corresponding author: Dr Zahra Najmi, zahranaj@yahoo.com}

1. Endometriosis Research Center, Rasoul-e-Akram Hospital. Iran University of Medical Sciences, Tehran, Iran.

2. Shahid Akbar-abadi Hospital, Iran University of Medical Sciences, Tehran, Iran.

3. Zanjan University of Medical Sciences, Zanjan, Iran.

4. Rasoul-e-Akram Hospital, Iran University of Medical Sciences, Tehran, Iran.

5. Pars Advanced and Minimally Invasive Medical Manners Research Center, Pars Hospital, Iran University of Medical Sciences, Tehran, Iran. tality and morbidity, multiple strategies have been introduced to improve the neonatal outcome by postponing the delivery $(5,6)$.

Tocolytic agents are the primary therapeutic options for TPL. For decades, $\beta$ agonists and magnesium sulfate have been used for the treatment of preterm labor. Recent studies have shown that nifedipine, a calcium-channel blocker, is a

$\uparrow$ What is "already known" in this topic:

Progesterone is a key hormone in the process of pregnancy. Previous studies have demonstrated the effectiveness of prophylactic progesterone for preterm Labor.

$\rightarrow$ What this article adds:

Intramuscular progesterone is as effective as oral nifedipine in treating threatened preterm labor. 
more effective and better tolerated tocolytic agent compared to traditional drugs $(7,8)$. Nifedipine is now the first choice for the suppression of preterm labor (9). However the use of nifedipine is associated with some side effects such as a headache, dizziness, flushing, and peripheral edema, and is contraindicated in women with hypotension, congestive heart failure, and aortic stenosis. There are also some concerns about its adverse effect on fetus (10).

Progesterone is a key hormone in the process of pregnancy, since its decline has been implicated in the initiation of labor (11). Previous studies have demonstrated the effectiveness of prophylactic progesterone for preterm labor in high-risk women (12). Prophylactic progesterone significantly reduces the risk of preterm birth, low birth weight, and neonatal complications (13). Based on these findings, progesterone might be used as a tocolytic agent in women with TPL. In this study, we evaluate this hypothesis and compare the tocolytic properties of progesterone with the standard treatment in our center.

\section{Methods}

\section{Subjects and Procedure}

This randomized controlled trial was carried out in a teaching hospital (Shahid Akbarabadi) in Tehran, Iran, from December 2011 to November 2012. The trial was approved by the Ethical Committee of Tehran University of Medical Sciences (code of ethical Committee is 338). The participants provided written informed consent. The IRCT registration is IRCT201112198469N1.

The study population was singleton pregnant women aged $>18$ years old with the diagnosis of TPL in 26-34 weeks' gestation. Threatened preterm labor was defined as spontaneous uterine contractions (at least 3 in 20 minutes). We excluded women with cervical dilatation $\geq 3 \mathrm{~cm}$, vaginal bleeding, vaginal discharge, uterine overdistention (due to polyhydramnios or multiple gestations), systemic infection, fever $>38^{\prime} \mathrm{C}$, fetal distress, intrauterine growth restriction, blood pressure $\geq 140 / 90$ and $\leq 100 / 60 \mathrm{mmHg}$, any obstetric contraindications to tocolytic agents, and history of any thromboembolic disorders. The primary outcome measure was the tocolytic efficacy of the two drugs (Nifedipine and progesterone).

Sample size calculations were based on a previous trial (14) which reported the prevention of preterm labor of $36.3 \%$ and $54.9 \%$ in the progesterone and placebo groups, respectively. Considering those rates, and with $\alpha=0.05$ and power $=80 \%$, a sample size of 150 women in each group was calculated.

On admission, all women were given one-hour bed rest while receiving $500 \mathrm{ml}$ of Ringer lactate and $50 \mathrm{mg}$ Pethidine (Meperidine hydrochloride, Dolantin). Patients, whose symptoms still remained, were randomly assigned to receive either oral nifedipine (Adalat capsules, Bayer $\mathrm{AG}$ ) or intramuscular progesterone (Prontogest, AMSA, Italy). We randomly allocate our cases by Excel software using Rand Between order. Allocation into each group was based on opening sealed and opaque envelopes indicating the drug name. Clinicians were not blinded to the treatment allocation.

Nifedipine was started with a loading dose of $30 \mathrm{mg}$ and continued with 10-20 mg every 4-6 hours until the pain relief. Nifedipine was discontinued if blood pressure reached $<90 / 50 \mathrm{~mm} \mathrm{Hg}$. Tocolysis was provided with $200 \mathrm{mg}$ of intramuscular progesterone $(100 \mathrm{mg}$ in each gluteus maximus muscle) in the second group. Considering known effect of nifedipine in inhibiting contractions, patients with continued uterine contractions after 3 hours of progesterone initiation were treated with nifedipine, using the same protocol. There is no difference regarding the price and availability of progesterone vials or nifedipine tablets in our country.

A course of intramuscular betamethasone was administered in all patients in both groups for promotion of fetal lung maturity. The uterine contractions were carefully monitored for the next 24 hours. If no contraction was encountered, the patient was discharged home. Patients were asked to limit their physical activity and alarmed of the signs and symptoms of preterm labor. Weekly perinatal visits were scheduled until the delivery.

\section{Study outcomes}

The primary outcome measure was the tocolytic efficacy of the two drugs. Other outcomes of interest were the gestational age at delivery, type of delivery, birth weight, admission to the neonatal intensive care unit (NICU), and duration of NICU and hospital stays.

\section{Statistical analysis}

Statistical analysis was performed using SPSS version 19. Baseline characteristics between two groups were compared using the Student's t-test and the Mann-Whitney test. To compare maternal and neonatal outcomes, Chi-square and the Student's t-test were used. Results are presented as frequency distribution or mean \pm standard deviation. Logistic regression was used to adjust the effect of the confounders. A p-value less than 0.05 was considered statistically significant.

\section{Results}

In total, 497 women were assessed for eligibility, of which 315 (63\%) women were randomized to receive either intra-muscular (IM) progesterone $(n=159)$ or oral Nifedipine $(n=156)$.

Women in both groups were statistically the same considering maternal age, gestational age, BMI, gravidity, parity, history of preterm birth, Bishop Score, and force of uterine contractions (Montevideo units) (Table 1).

Successful tocolysis was achieved in $83 \%$ and $82.7 \%$ of patients who received progesterone and nifedipine, respectively.

For 27 women in progesterone group whose contractions were continued, the second line protocol (Nifedipine) was started. Eighteen patients responded to second therapy, and other 9 progressed to delivery. In oral Nifedipine group all 26 patients whose contractions were continued progressed to delivery. 
Table 1. Baseline characteristics of two groups (Chi 2 test and T test)

\begin{tabular}{|c|c|c|c|c|c|}
\hline \multirow[t]{2}{*}{ Variable } & \multirow{2}{*}{$\begin{array}{l}\text { Progesterone } \\
\quad(n=159)\end{array}$} & \multirow{2}{*}{$\begin{array}{l}\text { Nifedipine } \\
(\mathrm{n}=156)\end{array}$} & \multirow[b]{2}{*}{$\mathrm{p}$} & \multicolumn{2}{|c|}{ CI $95 \%$} \\
\hline & & & & Lower & Upper \\
\hline Maternal age (years), mean \pm SD & $25.05 \pm 5.38$ & $25.25 \pm 4.61$ & 0.73 & -1.30 & 0.91 \\
\hline Gestational age of admission (days), mean \pm SD & $231.59 \pm 19.03$ & $231.22 \pm 12.23$ & 0.83 & -3.23 & 3.99 \\
\hline $\mathrm{BMI}\left(\mathrm{kg} / \mathrm{m}^{2}\right)$, mean $\pm \mathrm{SD}$ & $26.62 \pm 5.23$ & $26.14 \pm 4.84$ & 0.42 & -0.69 & 1.65 \\
\hline Gravidity, n (\%) & $1.60 \pm 0.92$ & $1.65 \pm 0.94$ & 0.63 & -0.25 & 0.15 \\
\hline 1 & $102(64.2 \%)$ & $93(59.6 \%)$ & & & \\
\hline 2 & $27(17.0 \%)$ & $36(23.1 \%)$ & 0.30 & & \\
\hline 3 & $21(13.2 \%)$ & $15(9.6 \%)$ & & & \\
\hline 4 & $9(5.7 \%)$ & $12(7.7 \%)$ & & & \\
\hline Parity, n (\%) & $0.43 \pm 068$ & $0.53 \pm 0.84$ & 0.23 & -0.27 & 0.66 \\
\hline 0 & $108(67.9 \%)$ & $99(63.5 \%)$ & & & \\
\hline 1 & $33(20.8 \%)$ & $39(25 \%)$ & 0.15 & & \\
\hline 2 & $18(11.3 \%)$ & $9(5.8 \%)$ & & & \\
\hline 3 & 0 & $9(5.8 \%)$ & & & \\
\hline History of Preterm Birth & $9(8.8 \%)$ & $6(5.4 \%)$ & 0.33 & -0.21 & 0.87 \\
\hline BISHOP score, mean \pm SD & $1.25 \pm 0.81$ & $1.27 \pm 0.91$ & 0.16 & -0.46 & 0.07 \\
\hline Uterine contractions (Montevideo) & $90.94 \pm 42.15$ & $82.59 \pm 42.64$ & 0.08 & -1.05 & 17.74 \\
\hline \multirow[t]{2}{*}{ Variable } & Progesterone & Nifedipine & & \multicolumn{2}{|c|}{ CI $95 \%$} \\
\hline & $(\mathrm{n}=102)$ & $(\mathrm{n}=111)$ & $\mathrm{p}$ & Lower & Upper \\
\hline Gestational age of delivery (days), mean \pm SD & $257.63 \pm 23.60$ & $256.62 \pm 14.37$ & 0.71 & -4.35 & 6.36 \\
\hline Gestational age, $\mathrm{n}(\%)$ & & & 0.50 & 0.57 & 0.29 \\
\hline Term & $68(67.3 \%)$ & $64(60.4 \%)$ & & & \\
\hline Preterm & $33(32.7 \%)$ & $42(39.6 \%)$ & & & \\
\hline Mode of delivery, n (\%) & & & 0.22 & 0.21 & 0.23 \\
\hline Vaginal & $56(54.9 \%)$ & $66(59.5 \%)$ & & & \\
\hline Cesarean & $46(45.1 \%)$ & $45(40.5 \%)$ & & & \\
\hline Delivery, n (\%) & & & 0.21 & 0.20 & 0.22 \\
\hline Within $48 \mathrm{~h}$ & $18(18.2 \%)$ & $15(14.3 \%)$ & & & \\
\hline Within 7 days & $3(3 \%)$ & $9(8.6 \%)$ & & & \\
\hline After 7 days & $78(78.8 \%)$ & $81(77.1 \%)$ & & & \\
\hline $\begin{array}{l}\text { Interval between intervention and delivery } \\
\text { (days), mean } \pm \mathrm{SD}\end{array}$ & $28.55 \pm 21.53$ & $23.65 \pm 13.64$ & 0.05 & -0.04 & 9.84 \\
\hline Birth weight (gr), mean \pm SD & $2980.00 \pm 529.79$ & $2812.57 \pm 711.43$ & 0.05 & -4.85 & 339.71 \\
\hline NICU admission, n (\%) & $0.33 \pm 0.76$ & $1.50 \pm 0.15$ & 0.00 & -1.80 & -0.52 \\
\hline NICU Stay (days), mean \pm SD & $0.33 \pm 0.77$ & $1.5 \pm 3.2$ & 0.10 & -0.23 & 0.02 \\
\hline Hospital Stay (days), mean \pm SD & $1.54 \pm 0.96$ & $2.05 \pm 1.43$ & 0.05 & -1.03 & $<0.001$ \\
\hline
\end{tabular}

We followed participants up to delivery for maternal and neonatal outcomes. Thirty women in the progesterone group and 18 women in the nifedipine group were lost to follow-up. Therefore 102 women in the progesterone group and 111 women in the nifedipine group were followed up to delivery (Fig. 1).

There was no significant difference between the two groups with regard to gestational age at delivery and type of delivery. Additionally, the numbers of patients who delivered within 48 hours, during the first week, and after the first week were statistically the same in both groups. The mean \pm SD time interval between the intervention and delivery in the progesterone-treated group and the nifedipine- treated group were $28.6 \pm 22$ days and $23.6 \pm 14$ days, respectively $(\mathrm{p}>0.05)$.

Despite the insignificant difference between the two groups in the proportion of NICU admission, the duration of NICU stay was significantly longer in the nifedipinetreated group. The difference in the neonatal birth weight and duration of antenatal hospital stay between the two groups was insignificant (Table 2).

Only one patient in the nifedipine group reached blood pressure of $70 / 40$ who responded to the drug discontinuation. This patient was excluded from the study. Minor side effects of nifedipine including headache, dizziness and constipation were recorded in $26 \%$ of patients. No side effect of IM progesterone including cellulitis and abscess

\begin{tabular}{|c|c|c|c|c|}
\hline Variable & OR Unadjusted & $\bar{p}$ & OR Adjusted & $\mathrm{p}$ \\
\hline BMI & $1.00(0.73,1.19)$ & 0.170 & $1.04(0.94-1.15)$ & 0.378 \\
\hline Gravidity & $0.94(0.14-1.70)$ & 0.951 & $0.44(0.10-2.00)$ & 0.295 \\
\hline Nulli Parity & $1.94(0.04-4.47)$ & 0.321 & $3.15(0.72-13.82)$ & 0.128 \\
\hline Gestational Age & $1.00(0.97-1.01)$ & 0.425 & $0.97(0.94-1.01)$ & 0.221 \\
\hline BISHOP Score & $1.91(1.95-2.05)$ & 0.201 & $0.94(0.56-1.56)$ & 0.813 \\
\hline Progesterone group* & $0.95(0.81-1.10)$ & 0.501 & $0.58(0.23-1.46)$ & 0.252 \\
\hline Fetus Weight & $1.72(1.05-2.35)$ & 0.001 & $1.00(1.01-1.02)$ & $<0.001$ \\
\hline
\end{tabular}

*Reference group: Nifedipine 


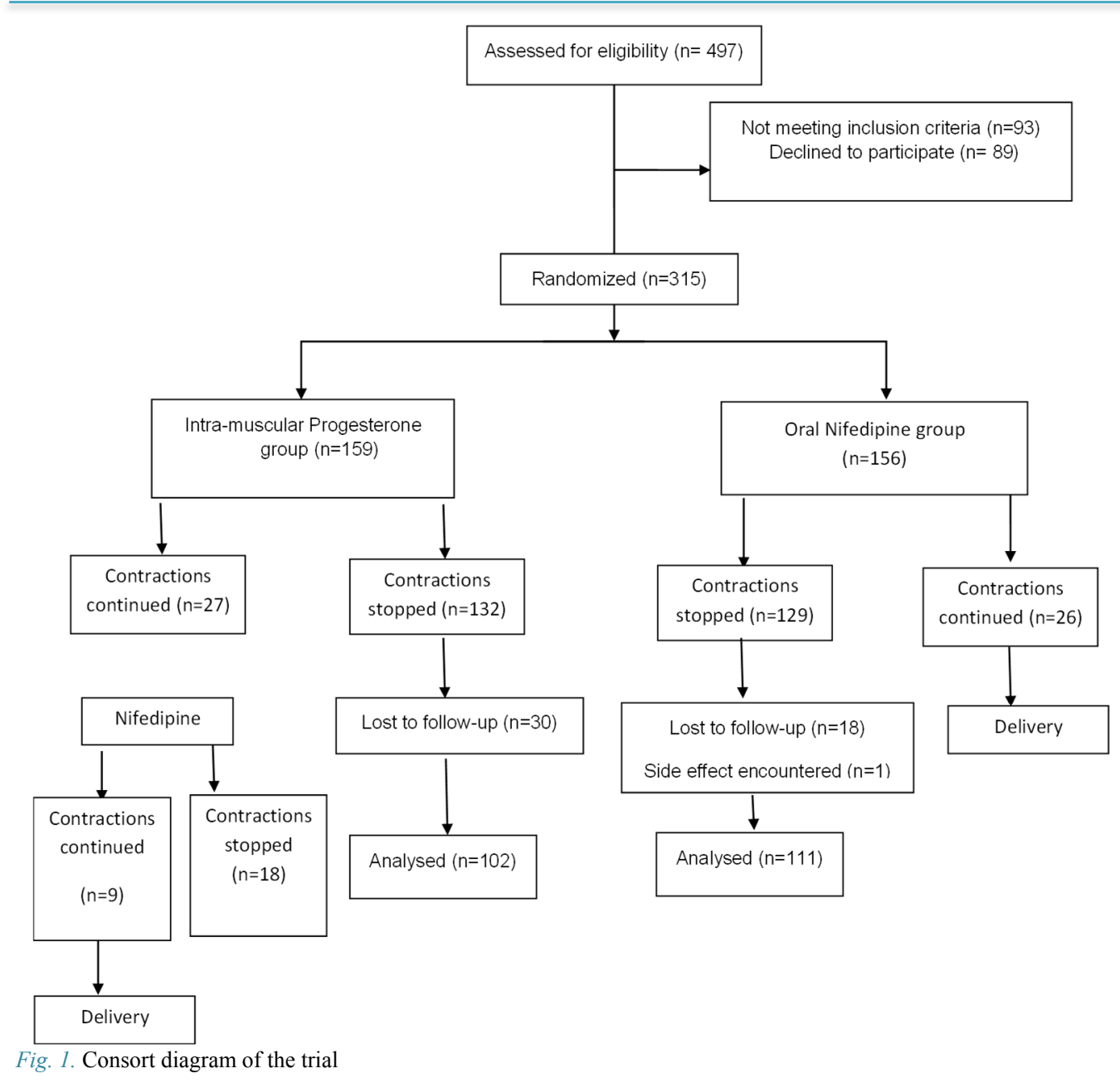

formation at the injection site was recorded, although the post-injection muscular pain was a common complaint $(32 \%)$.

The results of logistic regression analysis adjusted for probable confounders like BMI, gravidity, parity, gestational age, BISHOP score, and fetus weight showed no significant difference between groups (Table 3).

\section{Discussion}

In this randomized clinical trial, we compared the efficacy of IM progesterone with oral nifedipine in the treatment of women with TPL. Both drugs successfully inhibited uterine contractions. A single shot of progesterone comparably improved the maternal and neonatal outcomes. It also significantly reduced the duration of NICU stay as compared with oral nifedipine. None of the two drugs caused any major side effects.

The essential role of progesterone in the pregnancy maintenance has long been recognized (15). Progesterone promotes quiescence of the uterus and inhibits cervical ripening through its antagonizing actions on both cyclooxygenase-2 (COX-2) and inducible nitric oxide synthase (iNOS). Its immune-suppressive effect at feto-maternal interface protects decidual and chorion cells from induced apoptosis (16). It also interferes with the action of cortisol to change the expression of genes regulating the timing of labor (17).
Since its introduction in the mid 1930s, different progesterone formulations have been used in a wide variety of obstetrics situations (18). There is extensive evidence that progesterone might be successfully used in the treatment of threatened miscarriage (19), recurrent miscarriage $(20,21)$, and in women at high risk for preterm birth $(12,13)$. Although it has been stated that progesterone might not be an effective tocolytic when the process of labor (gap junctions formation and inflammatory cascade) has initiated (22), recent studies have reported a significant therapeutic role for progesterone.

Borna and Sahabi (23) evaluated the efficacy of maintenance tocolytic therapy with vaginal progesterone after successful inhibition of preterm labor in low-risk singleton women. Progesterone significantly improved pregnancy duration, respiratory distress syndrome rate, and low birth weight rate.

In another study by Facchinetti et al. (24) singleton pregnant women with TPL were received IM progesterone twice a week or observation after successful tocolysis with atosiban. Treatment with progesterone was associated with lower risk of cervical shortening and preterm birth.

Chawanpaiboon et al. (25) carried out a study to compare the efficacy of nifedipine, progesterone, and bed rest for inhibiting uterine contraction in TPL. All three interventions were comparably successful in inhibiting the contractions; however, nifedipine had the shortest onset of action. 
A recent Cochrane review analyzed the effectiveness of progesterone in the treatment of TPL (26). Progesterone use was associated with decreased shortening of cervical length and uterine contractions. However, because of limited available studies, the results were poorly attributable.

Although both drugs were effective in the treatment of TPL, our results showed that in progesterone arm, receiving the second line Nifedipine helped in better tocolytic effect. However, reports showed that a combination therapy had not significantly prolonged the pregnancy or improved its outcomes (27).

On the other hand, there is no agreement on the therapeutic route, formulation or dosage of progesterone yet (28). Vaginal administration of progesterone might increase the rate of PTL via repeated digital self-examination (29). The safety of progesterone is another field of concern. Progesterone may cause maternal side-effects such as headache, nausea, breast tenderness or coughing, but its administration has not been associated with congenital anomalies (30).

Our study had some limitations. Firstly, only singleton pregnancies were studied. As a result, our findings can be only interpreted in low-risk women for TPL. Secondly, the analyses might have limited power to detect differences between the two groups mostly due to a small number of included patients. Thirdly, the clinicians were not blinded to the treatment and finally high rate of our patients did not continue their follow-up.

\section{Conclusion}

In conclusion, single-dose progesterone injection has similar therapeutic efficacy to oral nifedipine in postponing the TPL. Its use was not associated with major maternal and neonatal complication. Future studies are needed to evaluate this treatment in larger populations.

\section{Conflict of Interests}

The authors declare that they have no competing interests.

\section{References}

1. McPheeters ML, Miller WC, Hartmann KE, Savitz DA, Kaufman JS, Garrett JM, et al. The epidemiology of threatened preterm labor: a prospective cohort study. Am J Obstet Gynecol. 2005;192(4):1325-9; discussion 9-30.

2. Vahdat M, Mehdizadeh A, Sariri E, Chaichian S, Najmi Z, Kadivar M. Placenta percreta invading broad ligament and parametrium in a woman with two previous cesarean sections: a case report. Case Rep Obstet Gynecol. 2012;2012.

3. Hassani V, Pazouki A, Nikoubakht N, Chaichian S, Sayarifard A, Khankandi AS. The effect of gabapentin on reducing pain after laparoscopic gastric bypass surgery in patients with morbid obesity: a randomized clinical trial. Anesthesio Pain Med. 2015;5(1).

4. Beck S, Wojdyla D, Say L, Betran AP, Merialdi M, Requejo JH, et al. The worldwide incidence of preterm birth: a systematic review of maternal mortality and morbidity. Bull World Health Organ. 2010;88(1):31-8.

5. Haram K, Mortensen JH, Wollen AL. Preterm delivery: an overview. Acta Obstet Gynecol Scand. 2003;82(8):687-704.

6. Vahdat M, Sariri E, Kashanian M, Najmi Z, Mobasseri A, Marashi M, et al. Can combination of hysterosalpingography and ultrasound replace hysteroscopy in diagnosis of uterine malformations in infertile women? MJIRI. 2016;30(1):364-7.

7. Conde-Agudelo A, Romero R, Kusanovic JP. Nifedipine in the management of preterm labor: a systematic review and metaanalysis. Am J Obstet Gynecol. 2011;204(2):134 e1-20.

8. King JF, Flenady V, Papatsonis D, Dekker G, Carbonne B. Calcium channel blockers for inhibiting preterm labour; a systematic review of the evidence and a protocol for administration of nifedipine. Aust NZ J Obstet Gynaecol. 2003;43(3):192-8.

9. Oei SG, Mol BW, de Kleine MJ, Brolmann HA. Nifedipine versus ritodrine for suppression of preterm labor; a meta-analysis. Acta Obstet Gynecol Scand. 1999;78(9):783-8.

10. Goldenberg RL. The management of preterm labor. Obstet Gynecol. 2002;100(5 Pt 1):1020-37.

11. Mitchell MD. The initiation of parturition. Current Obstet Gynaecol. 1994;4(2):74-8

12. Mackenzie R, Walker M, Armson A, Hannah ME. Progesterone for the prevention of preterm birth among women at increased risk: a systematic review and meta-analysis of randomized controlled trials. Am J Obstet Gynecol. 2006;194(5):1234-42.

13. Dodd JM, Crowther CA, Cincotta R, Flenady V, Robinson JS. Progesterone supplementation for preventing preterm birth: a systematic review and meta-analysis. Acta Obstet Gynecol Scand. 2005;84(6):526-33.

14. Meis PJ, Klebanoff M, Thom E, Dombrowski MP, Sibai B, Moawad $\mathrm{AH}$, et al. Prevention of recurrent preterm delivery by 17 alphahydroxyprogesterone caproate. N Engl J Med. 2003;348(24):2379-85.

15. Swyer GI, Daley D. Progesterone implantation in habitual abortion Br Med J. 1953;1(4819):1073-7.

16. Ransom CE, Murtha AP. Progesterone for preterm birth prevention. Obstet Gynecol Clin North Am. 2012;39(1):1-16, vii.

17. Karalis K, Goodwin G, Majzoub JA. Cortisol blockade of progesterone: a possible molecular mechanism involved in the initiation of human labor. Nat Med. 1996;2(5):556-60.

18. Di Renzo GC, Mattei A, Gojnic M, Gerli S. Progesterone and pregnancy. Curr Opin Obstet Gynecol. 2005;17(6):598-600.

19. Wahabi HA, Fayed AA, Esmaeil SA, Al Zeidan RA. Progestogen for treating threatened miscarriage. Cochrane Database Syst Rev. 2011(12):CD005943.

20. Walch KT, Huber JC. Progesterone for recurrent miscarriage: truth and deceptions. Best Pract Res Clin Obstet Gynaecol. 2008;22(2):37589.

21. Nardo LG, Sallam HN. Progesterone supplementation to prevent recurrent miscarriage and to reduce implantation failure in assisted reproduction cycles. Reprod Biomed Online. 2006;13(1):47-57.

22. Meis PJ. 17 hydroxyprogesterone for the prevention of preterm delivery. Obstet Gynecol. 2005;105(5 Pt 1):1128-35.

23. Borna S, Sahabi N. Progesterone for maintenance tocolytic therapy after threatened preterm labour: a randomised controlled trial. Aust N Z J Obstet Gynaecol. 2008;48(1):58-63.

24. Facchinetti F, Paganelli S, Comitini G, Dante G, Volpe A. Cervical length changes during preterm cervical ripening: effects of 17-alphahydroxyprogesterone caproate. Am J Obstet Gynecol. 2007;196(5):453 e1-4; discussion 21.

25. Chawanpaiboon S, Pimol K, Sirisomboon R. Comparison of success rate of nifedipine, progesterone, and bed rest for inhibiting uterine contraction in threatened preterm labor. J Obstet Gynaecol Res. 2011;37(7):787-91

26. Su LL, Samuel M, Chong YS. Progestational agents for treating threatened or established preterm labour. Cochrane Database Syst Rev. 2010(1):CD006770.

27. Tan PC, King AS, Vallikkannu N, Omar SZ. Single dose 17 alphahydroxyprogesterone caproate in preterm labor: a randomized trial. Arch Gynecol Obstet. 2012;285(3):585-90.

28. Coomarasamy A, Thangaratinam S, Gee H, Khan KS. Progesterone for the prevention of preterm birth: a critical evaluation of evidence. Eur J Obstet Gynecol Reprod Biol. 2006;129(2):111-8.

29. Thornton JG. Progesterone and preterm labor--still no definite answers. N Engl J Med. 2007;357(5):499-501.

30. Dodd JM, Crowther CA. The role of progesterone in prevention of preterm birth. Int J Womens Health. 2010;1:73-84. 